\title{
New Iteration Methods for Time-Fractional Modified Nonlinear Kawahara Equation
}

\author{
Abdon Atangana, ${ }^{1}$ Necdet Bildik, ${ }^{2}$ and S. C. Oukouomi Noutchie ${ }^{3}$ \\ ${ }^{1}$ Institute for Groundwater Studies, Faculty of Natural and Agricultural Sciences, University of the Free State, \\ Bloemfontein 9301, South Africa \\ ${ }^{2}$ Department of Mathematics, Faculty of Art \& Sciences, Celal Bayar University, Muradiye Campus, 45047 Manisa, Turkey \\ ${ }^{3}$ Department of Mathematical Sciences, North-West University, Mafikeng Campus, Mmabatho 2735, South Africa
}

Correspondence should be addressed to Abdon Atangana; abdonatangana@yahoo.fr

Received 3 September 2013; Accepted 26 September 2013; Published 16 January 2014

Academic Editor: Adem Kiliçman

Copyright (C) 2014 Abdon Atangana et al. This is an open access article distributed under the Creative Commons Attribution License, which permits unrestricted use, distribution, and reproduction in any medium, provided the original work is properly cited.

We put side by side the methodology of two comparatively new analytical techniques to get to the bottom of the system of nonlinear fractional modified Kawahara equation. The technique is described and exemplified with a numerical example. The dependability of both methods and the lessening in computations give these methods a wider applicability. In addition, the computations implicated are very simple and undemanding.

\section{Introduction}

Within the scope of fractional calculus in the recent decade several scholars have modeled physical and engineering problems. Respective scholar while dealing with real world problems found out that it is worth describing these phenomena with the idea of derivatives with fractional order. While searching the literature, we found out that, this concept of noninteger order derivative not only has been intensively used but also has played an essential role in assorted branches of sciences including but not limited to hydrology, chemistry, image processing, electronics and mechanics; the applicability of this philosophy can be found in [1-10]. In the foregone respective decennial, the research of travelling-wave solutions for nonlinear equations has played a crucial character in the examination of nonlinear physical phenomena.

Nonlinear wave phenomena of dispersion, dissipation, diffusion, reaction, and convection are very important in nonlinear wave equations. Concepts like solitons, peakons, kinks, breathers, cusps, and compactons have now been thoroughly investigated in the scientific literature [11-13].
Various powerful mathematical methods such as the inverse scattering method, bilinear transformation [14], the tanhsech method [15, 16], extended tanh method [16], Expfunction method [17-19], sine-cosine method [20] Adomian decomposition method [21], Exp-function method [22], homotopy perturbation method [23] have been proposed for obtaining exact and approximate analytical solutions.

The purpose of this paper is to examine the approximated solution of the nonlinear fractional modified Kawahara equation, using the relatively new analytical method, the Homotopy decomposition method (HDM), and the Sumudu transform method. The fractional partial differential equations under investigation here are given below as

$$
\begin{gathered}
\partial_{t}^{\alpha} u(x, t)+u^{2}(x, t) u_{x}(x, t)+p u_{x x}(x, t) \\
+q u_{x x x}(x, t)=0, \quad 0<\alpha \leq 1
\end{gathered}
$$

subject to the initial condition

$$
u(x, 0)=\frac{3 p}{\sqrt{-10 q}} \operatorname{sech}[K x]^{2}, \quad K=\frac{1}{2} \sqrt{\frac{-p}{5 q}} .
$$


The outstanding of this paper is prearranged as follows. In Section 2 we present a succinct history of the fractional derivative order and their properties. We present the basic ideal of the HDM and the STM for solving high order nonlinear fractional partial differential equations. We present their application to fractional nonlinear differential equations (1) and (2) and numerical results in Section 4. The conclusions are then given in Section 5 .

\section{Fractional Derivative Order}

2.1. Brief History. There exists a vast literature on different definitions of fractional derivatives [24-27]. The most popular ones are the Riemann-Liouville and the Caputo derivatives. For Caputo we have

$$
{ }_{0}^{c} D_{x}^{\alpha}(f(x))=\frac{1}{\Gamma(n-\alpha)} \int_{0}^{x}(x-t)^{n-\alpha-1} \frac{d^{n} f(t)}{d t^{n}} d t .
$$

For the case of Riemann-Liouville we have the following definition:

$$
D_{x}^{\alpha}(f(x))=\frac{1}{\Gamma(n-\alpha)} \frac{d^{n}}{d x^{n}} \int_{0}^{x}(x-t)^{n-\alpha-1} f(t) d t
$$

Each fractional derivative presents some advantages and disadvantages [24-27], Jumarie (see [28, 29]) proposed a simple alternative definition to the Riemann-Liouville derivative.

\section{Consider}

$$
\begin{aligned}
D_{x}^{\alpha} & (f(x)) \\
& =\frac{1}{\Gamma(n-\alpha)} \frac{d^{n}}{d x^{n}} \int_{0}^{x}(x-t)^{n-\alpha-1}\{f(t)-f(0)\} d t .
\end{aligned}
$$

\subsection{Properties and Definitions}

Definition 1. A real function $f(x), x>0$, is said to be in the space $c_{\mu}, \mu \in \mathbb{R}$, if there exists a real number $p>\mu$, such that $f(x)=x^{p} h(x)$, where $h(x) \in C[0, \infty)$, and0020it is said to be in space $C_{\mu}^{m}$ if $f^{(m)} \in C_{\mu}, m \in \mathbb{N}$.

Definition 2. The Riemann-Liouville fractional integral operator of order $\alpha \geq 0$, of a function $f \in C_{\mu}, \mu \geq-1$, is defined as

$$
\begin{gathered}
J^{\alpha} f(x)=\frac{1}{\Gamma(\alpha)} \int_{0}^{x}(x-t)^{\alpha-1} f(t) d t, \quad \alpha>0, x>0, \\
J^{0} f(x)=f(x) .
\end{gathered}
$$

Properties of the operator can be found in [26, 27], we mention only the following.

$$
\begin{gathered}
\text { For } f \in C_{\mu}, \quad \mu \geq-1, \alpha, \beta \geq 0, \text { and } \gamma>-1 \text { : } \\
J^{\alpha} J^{\beta} f(x)=J^{\alpha+\beta} f(x), \quad J^{\alpha} J^{\beta} f(x)=J^{\beta} J^{\alpha} f(x), \\
J^{\alpha} x^{\gamma}=\frac{\Gamma(\gamma+1)}{\Gamma(\alpha+\gamma+1)} x^{\alpha+\gamma} .
\end{gathered}
$$

Lemma 3. If $m-1<\alpha \leq m, m \in \mathbb{N}$ and $f \in C_{\mu}^{m}, \mu \geq-1$, then

$$
\begin{gathered}
D^{\alpha} J^{\alpha} f(x)=f(x), \\
J^{\alpha} D_{0}^{\alpha} f(x)=f(x)-\sum_{k=0}^{m-1} f^{(k)}\left(0^{+}\right) \frac{x^{k}}{k !} \\
x>0 .
\end{gathered}
$$

Definition 4 (partial derivatives of fractional order). Assume now that $f(\mathbf{x})$ is a function of $n$ variables, $x_{i}, i=1, \ldots, n$, also of class $C$ on $D \in \mathbb{R}_{n}$. As an extension of Definition 3 we define partial derivative of order $\alpha$ for $f(x)$ with respect to $x_{i}$ using the function.

$$
a \partial_{\underline{\mathbf{x}}}^{\alpha} f=\left.\frac{1}{\Gamma(m-\alpha)} \int_{a}^{x_{i}}\left(x_{i}-t\right)^{m-\alpha-1} \partial_{x_{i}}^{m} f\left(x_{j}\right)\right|_{x_{j}=t} d t .
$$

If it exists, where $\partial_{x_{i}}^{m}$ is the usual partial derivative of integer order $m$.

\section{Basic Information Regarding the Methodology of the HDM [32-35]}

To illustrate the basic idea of this method we consider a general nonlinear nonhomogeneous fractional $p$ differential equation with initial conditions of the following form [30]

$$
\frac{\partial^{\alpha} V(x, t)}{\partial t^{\alpha}}=L(V(x, t))+N(V(x, t))+g(t), \quad \alpha>0
$$

subject to the initial condition

$$
\begin{gathered}
D_{0}^{\alpha-k} U(x, 0)=f_{k}, \quad(k=0, \ldots, n-1), \\
D_{0}^{\alpha-n} V(x, 0)=0, \quad n=[\alpha], \\
D_{0}^{k} U(x, 0)=h_{k}, \quad(k=0, \ldots, n-1), \\
D_{0}^{n} V(x, 0)=0, \quad n=[\alpha],
\end{gathered}
$$

where, $\partial^{\alpha} / \partial t^{\alpha}$ denotes the Caputo or Riemann-Liouville fraction derivative operator, $g$ is a known function, $N$ is the general nonlinear fractional differential operator and $L$ represents a linear fractional differential operator [30]. The method first step here is to transform the fractional partial differential equation to the fractional partial integral equation by applying the inverse operator $\partial^{\alpha} / \partial t^{\alpha}$ on both sides of (10) to obtain the following: In the case of Riemann-Liouville fractional derivative,

$$
\begin{aligned}
V(x, t)= & \sum_{j=1}^{n-1} \frac{g_{j}(x)}{\Gamma(\alpha-j+1)} t^{\alpha-j} \\
& +\frac{1}{\Gamma(\alpha)} \int_{0}^{t}(t-\tau)^{\alpha-1}[L(V(x, \tau))+N(V(x, \tau)) \\
& +g(x, \tau)] d \tau .
\end{aligned}
$$


In the case of Caputo fractional derivative,

$$
\begin{aligned}
U(x, t)= & \sum_{j=1}^{n-1} \frac{h_{j}(x)}{\Gamma(\alpha-j+1)} t^{j} \\
& +\frac{1}{\Gamma(\alpha)} \int_{0}^{t}(t-\tau)^{\alpha-1}[L(V(x, \tau))+N(V(x, \tau)) \\
& +g(x, \tau)] d \tau .
\end{aligned}
$$

Or in general by putting

$$
\sum_{j=1}^{n-1} \frac{f_{j}(x)}{\Gamma(\alpha-j+1)} t^{\alpha-j}=f(x, t)
$$

or

$$
f(x, t)=\sum_{j=1}^{n-1} \frac{g_{j}(x)}{\Gamma(\alpha-j+1)} t^{j} .
$$

We obtain

$$
\begin{aligned}
V(x, t)= & T(x, t) \\
+\frac{1}{\Gamma(\alpha)} \int_{0}^{t}(t-\tau)^{\alpha-1} & {[L(V(x, \tau))+N(V(x, \tau))} \\
& +g(x, \tau)] d \tau .
\end{aligned}
$$

In the homotopy decomposition method, the basic assumption is that the solutions can be written as a power series in $p$ [30]

$$
\begin{gathered}
V(x, t, p)=\sum_{n=0}^{\infty} p^{n} U_{n}(x, t), \\
V(x, t)=\lim _{p \rightarrow 1} V(x, t, p),
\end{gathered}
$$

and the nonlinear term can be decomposed as

$$
N V(x, t)=\sum_{n=0}^{\infty} p^{n} \mathscr{H}_{n}(V)
$$

where $p \in(0,1]$ is an embedding parameter. $\mathscr{H}_{n}(U)$ is the He's polynomials that can be generated by

$$
\begin{array}{r}
\mathscr{H}_{n}\left(V_{0}, \ldots, V_{n}\right)=\frac{1}{n !} \frac{\partial^{n}}{\partial p^{n}}\left[N\left(\sum_{j=0}^{\infty} p^{j} V_{j}(x, t)\right)\right], \\
n=0,1,2, \ldots
\end{array}
$$

The homotopy decomposition method is obtained by the combination of homotopy technique with Abel integral and is given by [30]

$$
\begin{aligned}
\sum_{n=0}^{\infty} p^{n} V_{n}(x, t)-F(x, t) & \\
=\frac{p}{\Gamma(\alpha)} \int_{0}^{t}(t-\tau)^{\alpha-1} & {\left[g(x, \tau)+L\left(\sum_{n=0}^{\infty} p^{n} V_{n}(x, \tau)\right)\right.} \\
& \left.+N\left(\sum_{n=0}^{\infty} p^{n} V_{n}(x, \tau)\right)\right] d \tau .
\end{aligned}
$$

Comparing the terms of same powers of $p$ gives solutions of various orders with the first term:

$$
V_{0}(x, t)=F(x, t) .
$$

Theorem 5 (see [31]). Assuming that $X \times T \subset \mathbb{R} \times \mathbb{R}^{+}$is a Banach space with a well-defined norm \|\| , over which the series sequence of the approximate solution of (10) is defined, and the operator $G\left(U_{n}(x, t)\right)=U_{n+1}(x, t)$ defining the series solution of (14) satisfies the Lipschitzian conditions, that is, $\left\|G\left(U_{k}{ }^{*}\right)-G\left(U_{k}\right)\right\| \leq \varepsilon\left\|U^{*}{ }_{k}(x, t)-U_{k}(x, t)\right\|$ for all $(x, t, k) \epsilon$ $X \times T \times \mathbb{N}$, then series solution obtained (17) is unique.

Proof (see [31]). Assume that $U(x, t)$ and $U^{*}(x, t)$ are the series solution satisfying (10); then

$$
U^{*}(x, t, p)=\sum_{n=0}^{\infty} p^{n} U^{*}{ }_{n}(x, t),
$$

with initial guess $T(x, t)$

$$
U(x, t, p)=\sum_{n=0}^{\infty} p^{n} U_{n}(x, t)
$$

also with initial guess $T(x, t)$ therefore

$$
\left\|U_{n}^{*}(x, t)-U_{n}(x, t)\right\|=0, \quad n=0,1,2, \ldots
$$

By the recurrence for $n=0, U^{*}{ }_{n}(x, t)=U_{n}(x, t)=$ $T(x, t)$, assume that for $n>k \geq 0,\left\|U^{*}{ }_{k}(x, t)-U_{k}(x, t)\right\|=0$. Then

$$
\begin{aligned}
\left\|U^{*}{ }_{k+1}(x, t)-U_{k+1}(x, t)\right\| & =\left\|G\left(U_{k}^{*}\right)-G\left(U_{k}\right)\right\| \\
& \leq \varepsilon\left\|U^{*}{ }_{k}(x, t)-U_{k}(x, t)\right\|=0,
\end{aligned}
$$

which completes the proof.

\section{Background of Sumudu Transform}

Definition 6 (see [34]). The Sumudu transform of a function $f(t)$, defined for all real numbers $t \geq 0$, is the function $F_{s}(u)$, defined by

$$
S(f(t))=F_{s}(u)=\int_{0}^{\infty} \frac{1}{u} \exp \left[-\frac{t}{u}\right] f(t) d t .
$$


Theorem 7 (see [35]). Let $G(u)$ be the Sumudu transform of $f(t)$ such that

(i) $G(1 / s) / s$ is a meromorphic function, with singularities having $\operatorname{Re}[s] \leq \gamma$,

(ii) there exists a circular region $\Gamma$ with radius $R$ and positive constants $M$ and $K$ with $|G(1 / s) / s|<M R^{-K}$; then the function $f(t)$ is given by

$$
\begin{aligned}
S^{-1}(G(s)) & =\frac{1}{2 \pi i} \int_{\gamma-i \infty}^{\gamma+i \infty} \exp [s t] G\left(\frac{1}{s}\right) \frac{d s}{s} \\
& =\sum \text { residual }\left[\exp [s t] \frac{G(1 / s)}{s}\right] .
\end{aligned}
$$

For the proof see [36].

4.1. Basics of the Sumudu Transform Method. We illustrate the basic idea of this method [34-41], by considering a general fractional nonlinear nonhomogeneous partial differential equation with the initial condition of the form

$$
D_{t}^{\alpha} U(x, t)=L(U(x, t))+N(U(x, t))+f(x, t), \quad \alpha>0,
$$

subject to the initial condition

$$
\begin{gathered}
D_{0}^{k} U(x, 0)=g_{k}, \quad(k=0, \ldots, n-1), \\
D_{0}^{n} U(x, 0)=0, \quad n=[\alpha],
\end{gathered}
$$

where $D_{t}^{\alpha}$ denotes without loss of generality the Caputo fraction derivative operator, $f$ is a known function, $N$ is the general nonlinear fractional differential operator and $L$ represents a linear fractional differential operator. obtain

Applying the Sumudu transform on both sides of (29), we

$$
\begin{aligned}
S\left[D_{t}^{\alpha} U(x, t)\right]= & S[L(U(x, t))]+S[N(U(x, t))] \\
& +S[f(x, t)] .
\end{aligned}
$$

Using the property of the Sumudu transform, we have

$$
\begin{aligned}
S[U(x, t)]= & u^{\alpha} S[L(U(x, t))]+u^{\alpha} S[N(U(x, t))] \\
& +u^{\alpha} S[f(x, t)]+g(x, t) .
\end{aligned}
$$

Now applying the Sumudu inverse on both sides of (19) we obtain

$$
\begin{aligned}
U(x, t)= & S^{-1}\left[u^{\alpha} S[L(U(x, t))]+u^{\alpha} S[N(U(x, t))]\right] \\
& +G(x, t)
\end{aligned}
$$

$G(x, t)$ represents the term arising from the known function $f(x, t)$ and the initial conditions.

Now we apply the HPM:

$$
U(x, t)=\sum_{n=0}^{\infty} p^{n} U_{n}(x, t) .
$$

The nonlinear term can be decomposed as follows:

$$
N U(x, t)=\sum_{n=0}^{\infty} p^{n} \mathscr{H}_{n}(U)
$$

using the He's polynomial $\mathscr{H}_{n}(U)$ given as

$$
\begin{aligned}
& \mathscr{H}_{n}\left(U_{0}, \ldots, U_{n}\right) \\
& =\frac{1}{n !} \frac{\partial^{n}}{\partial p^{n}}\left[N\left(\sum_{j=0}^{\infty} p^{j} U_{j}(x, t)\right)\right], \quad n=0,1,2, \ldots
\end{aligned}
$$

Substituting (35) and (36),

$$
\begin{aligned}
& \sum_{n=0}^{\infty} p^{n} U_{n}(x, t)= G(x, t) \\
&+p\left[S ^ { - 1 } \left[u^{\alpha} S\left[L\left(\sum_{n=0}^{\infty} p^{n} U_{n}(x, t)\right)\right]\right.\right. \\
&\left.\left.+u^{\alpha} S\left[N\left(\sum_{n=0}^{\infty} p^{n} U_{n}(x, t)\right)\right]\right]\right],
\end{aligned}
$$

which is the coupling of the Sumudu transform and the HPM using He's polynomials. Comparing the coefficients of like powers of $p$, the following approximations are obtained:

$$
\begin{gathered}
p^{0}: U_{0}(x, t)=G(x, t), \\
p^{1}: U_{1}(x, t)=S^{-1}\left[u^{\alpha} S\left[L\left(U_{0}(x, t)\right)+H_{0}(U)\right]\right], \\
p^{2}: U_{2}(x, t)=S^{-1}\left[u^{\alpha} S\left[L\left(U_{1}(x, t)\right)+H_{1}(U)\right]\right], \\
p^{3}: U_{3}(x, t)=S^{-1}\left[u^{\alpha} S\left[L\left(U_{2}(x, t)\right)+H_{2}(U)\right]\right], \\
p^{n}: U_{n}(x, t)=S^{-1}\left[u^{\alpha} S\left[L\left(U_{n-1}(x, t)\right)+H_{n-1}(U)\right]\right] .
\end{gathered}
$$

Finally, we approximate the analytical solution $U(x, t)$ by truncated series

$$
U(x, t)=\lim _{N \rightarrow \infty} \sum_{n=0}^{N} U_{n}(x, t) .
$$

The above series solutions generally converge very rapidly. 


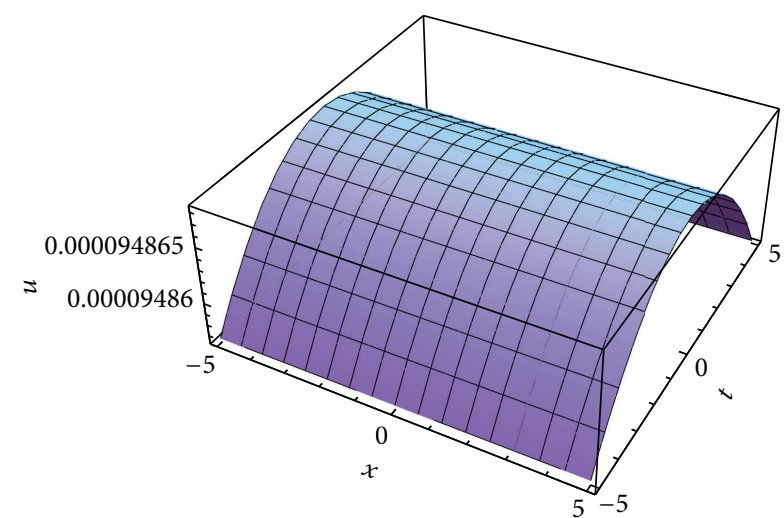

FIgURE 1: Approximated solutions for $\boldsymbol{\alpha}=0.45$.

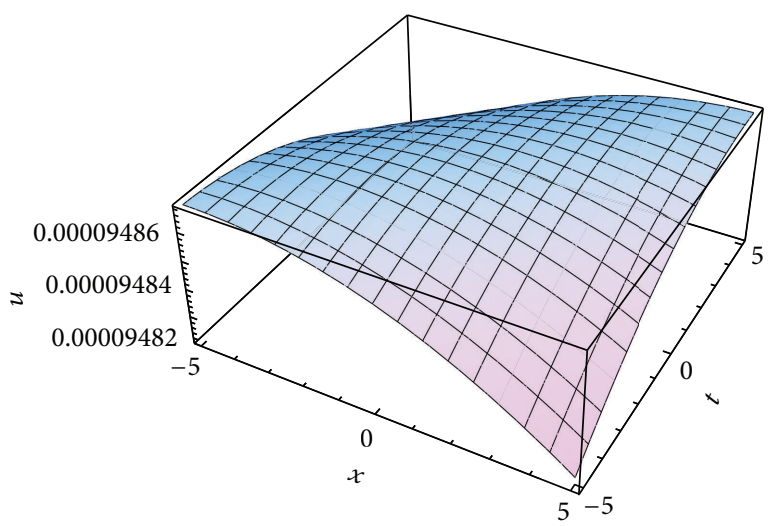

FIGURE 2: Approximated solution for $\boldsymbol{\alpha}=0.98$.

\section{Application}

5.1. Application with HDM. In this section we apply this method for solving nonlinear of fractional differential equation (1). Following the steps involve in the HDM, we arrive at the following equation:

$$
\begin{aligned}
& \sum_{n=0}^{\infty} p^{n} u_{n}(x, t) \\
& =u(x, 0) \\
& -\frac{p}{\Gamma(\alpha)} \int_{0}^{t}(t-\tau)^{\alpha-1} \\
& \times\left(\left(\sum_{n=0}^{\infty} p^{n} u_{n}(x, t)\right)^{2}\left(\sum_{n=0}^{\infty} p^{n} u_{n}(x, t)\right)_{x}\right. \\
& \quad+P\left(\sum_{n=0}^{\infty} p^{n} u_{n}(x, t)\right)_{x x} \\
& \left.\quad+q\left(\sum_{n=0}^{\infty} p^{n} u_{n}(x, t)\right)_{x x x x}\right) .
\end{aligned}
$$

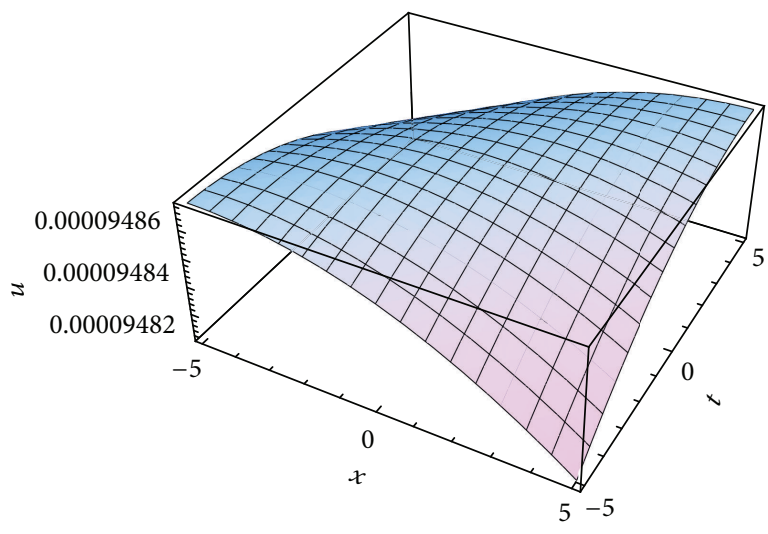

FIgURE 3: Exact solution for $\boldsymbol{\alpha}=\mathbf{1}$.

Now comparing the terms of the same power of $p$ we arrive at the following integral equations:

$$
\begin{gathered}
p^{0}, u_{0}(x, t)=u(x, 0), \quad u_{0}(x, 0)=u(x, 0), \\
p^{1}: u_{1}(x, t) \\
=-\frac{1}{\Gamma(\alpha)} \int_{0}^{t}(t-\tau)^{\alpha-1} \\
\quad \times\left(u_{0}^{2}\left(u_{0}\right)_{x}+P\left(u_{0}\right)_{x x}\right. \\
\left.\quad+q\left(u_{0}\right)_{x x x x}\right) d \tau, \quad u_{1}(x, 0)=0, \\
p^{n}: u_{n}(x, t) \quad-\frac{1}{\Gamma(\alpha)} \int_{0}^{t}(t-\tau)^{\alpha-1} \\
\times\left(\sum_{j=0}^{n-1} \sum_{k=0}^{j} u_{j} u_{j-k}\left(u_{n-j-1}\right)_{x}\right. \\
\left.+P\left(u_{n-1}\right)_{x x}+q\left(u_{n-1}\right)_{x x x x}\right) d \tau, \\
u_{n}(x, 0)=0, \quad n \geq 2 .
\end{gathered}
$$

The following solutions are straightforward obtained:

$$
u_{0}(x, t)=\frac{3 P}{\sqrt{-10 q}}(\operatorname{sech}(K x))^{2} .
$$


For simplicity we put $a=3 P / \sqrt{-10 q}$

$$
\begin{aligned}
& u_{1}(x, t) \\
& =\frac{3 P K t^{\alpha}(\operatorname{sech}(K x))^{2}}{4 \Gamma(1+\alpha) \sqrt{-10 q}} \\
& \quad \times\left(8 K\left(P-20 K^{2} q\right) \cosh (K x)\right. \\
& \quad+K\left(P+100 K^{2} q\right) \cosh (3 K x) \\
& \left.\quad-K\left(P+4 K^{2} q\right) \cosh (5 k x)+8 a^{2} \sinh (K x)\right),
\end{aligned}
$$$$
u_{2}(x, t)
$$$$
=\frac{1}{\Gamma^{2}(\alpha+1) \Gamma(0.5+\alpha) \Gamma(1+3 \alpha)}
$$$$
\times\left(2^{-5-2 \alpha} a K t^{\alpha}(\operatorname{sech}(K x))^{12}\right.
$$$$
\times(\Gamma(1+\alpha) \Gamma(1+3 \alpha)
$$$$
\times\left(4^{3+\alpha} a^{2}(\cosh (K x))^{5} \Gamma(0.5+\alpha) \sinh (K x)\right.
$$$$
+K \sqrt{\pi} t^{\alpha}\left(-64 a^{4}+276(K P)^{2}\right.
$$$$
-20832 K^{4} P q+1087296 K^{6} q^{2}
$$$$
+2\left(32 a^{4}+165(K P)^{2}-7224 K^{4} P q\right.
$$$$
\left.\left.-45360 K^{6} q^{2}\right) \cosh (2 K x)\right)
$$$$
-768 K^{4} q\left(-17 P+1240 K^{2} q\right) \cosh (4 K x)
$$$$
-75 K^{2} P^{2} \cosh (6 K x)
$$$$
+5736 K^{4} P q \cosh (6 K x)
$$$$
+217680 K^{6} q^{2} \cosh (6 K x)
$$$$
-20 K^{2} P^{2} \cosh (8 K x)
$$$$
-928 K^{4} P q \cosh (8 K x)
$$$$
-8000 K^{6} q^{2} \cosh (8 K x)
$$$$
+K^{2} P^{2} \cosh (10 K x)
$$$$
+8 K^{4} P q \cosh (10 K x)
$$$$
+16 K^{6} q^{2} \cosh (6 K x)
$$$$
+592 a^{2} K P \sinh (2 K x)
$$$$
-86720 a^{2} K^{3} q \sinh (2 K x)
$$$$
+176 a^{2} K P \sinh (4 K x)
$$$$
+35264 a^{2} K^{3} q \sinh (4 K x)
$$$$
-80 a^{2} K P \sinh (6 K x)
$$

$\left.-2624 a^{2} K^{3} q \sinh (6 K x)\right)$

$-2 a^{2} K^{2} t^{2 \alpha} \Gamma(0.5+\alpha)$

$\times \Gamma(1+2 \alpha)(\operatorname{sech}(K x))^{4}$

$\times\left(4^{3+\alpha} a^{4}-33 \times 2^{1+2 \alpha}(K P)^{2}+147\right.$

$$
\left.\times 4^{2+\alpha} K^{4} P q-1113 \times 2^{5+2 \alpha} K^{6} q^{2}\right)
$$$$
-2^{1+2 \alpha}\left(\left(32 a^{4}+39(K P)^{2}-744 K^{4} P q\right.\right.
$$

$$
\left.-36000 K^{6} q^{2}\right)
$$

$\times \cosh (2 K x)+3$

$\times 2^{9+2 \alpha} K^{4} q$

$\times\left(-P+20 K^{2} q\right) \cosh (4 K x)$

$+15 \times 4^{\alpha} K^{2} P^{2} \cosh (6 K x)-57$

$\times 2^{3+2 \alpha} K^{4} P q \cosh (6 K x)-705$

$\times 4^{2+\alpha} K^{6} q^{2} \cosh (6 K x)+2^{1+3 \alpha}$

$\times K^{2} P^{2} \cosh (8 K x)+13 \times 4^{2+\alpha}$

$\times K^{4} P q \cosh (8 K x)+25 \times 2^{5+2 \alpha}$

$\times K^{6} q^{2} \cosh (8 K x)$

$-4^{\alpha} K^{2} P^{2} \cosh (10 K x)$

$-2^{3+2 \alpha} K^{4} P q \cosh (10 K x)$

$-4^{2+\alpha} K^{6} q^{2} \cosh (10 K x)+7$

$\times 4^{2+\alpha} a^{2} K P \sinh (2 K x)+65$

$\times 4^{3+\alpha} a^{2} K^{3} q \sinh (2 K x)-2^{5+2 \alpha} a^{2}$

$\times K P \sinh (4 K x)-13 \times 2^{7+2 \alpha} a^{2} K^{3}$

$\times q \sinh (4 K x)+4^{2+\alpha} a^{2} K q \sinh (6 K x)$

$\left.\left.\left.+4^{3+\alpha} a^{2} K^{3} q \sinh (6 K x)\right) \tanh (K x)\right)\right)$,

and so on; using the package Mathematica, in the same manner one can obtain the rest of the components. But, here, few terms were computed and the asymptotic solution is given by

$$
\begin{aligned}
u(x, t)= & u_{0}(x, t)+u_{1}(x, t)+u_{2}(x, t) \\
& +u_{3}(x, t)+\cdots
\end{aligned}
$$

The figures show the graphical representation of the approximated solution of the system of nonlinear modified fractional Kawahara equation for $P=0.0001, q=-1$. The approximate 
solutions of main problem have been depicted in Figures 1, 2 , and 3 which were plotted in Mathematica according to different $\alpha$ values.

5.2. Applications with STM. In this subdivision, we take advantage of the line of attack of the Sumudu transform technique to obtain approximated solution of the adapted fractional Karawana equation. According to the steps involved in the Sumudu transfrom method, we arrive at the next series solution.

Consider

$$
\begin{aligned}
& u_{0}(x, t)=\frac{3 P}{\sqrt{-10 q}}(\operatorname{sech}(K x))^{2} \\
& u_{1}(x, t) \\
& =\frac{3 P K t^{\alpha}(\operatorname{sech}(K x))^{2}}{4 \Gamma(1+\alpha) \sqrt{-10 q}} \\
& \times\left(8 K\left(P-20 K^{2} q\right) \cosh (K x)\right. \\
& +K\left(P+100 K^{2} q\right) \cosh (3 K x) \\
& \left.-K\left(P+4 K^{2} q\right) \cosh (5 k x)+8 a^{2} \sinh (K x)\right), \\
& u_{2}(x, t) \\
& =\frac{1}{\Gamma^{2}(\alpha+1) \Gamma(0.5+\alpha) \Gamma(1+3 \alpha)} \\
& \times\left(2^{-5-2 \alpha} a K t^{\alpha}(\operatorname{sech}(K x))^{12}\right. \\
& \times(\Gamma(1+\alpha) \Gamma(1+3 \alpha) \\
& \times\left(4^{3+\alpha} a^{2}(\cosh (K x))^{5} \Gamma(0.5+\alpha) \sinh (K x)\right. \\
& +K \sqrt{\pi} t^{\alpha}\left(-64 a^{4}+276(K P)^{2}\right. \\
& -20832 K^{4} P q+1087296 K^{6} q^{2} \\
& +2\left(32 a^{4}+165(K P)^{2}-7224 K^{4} P q\right. \\
& \left.\left.-45360 K^{6} q^{2}\right) \cosh (2 K x)\right) \\
& -768 K^{4} q\left(-17 P+1240 K^{2} q\right) \cosh (4 K x) \\
& -75 K^{2} P^{2} \cosh (6 K x) \\
& +5736 K^{4} P q \cosh (6 K x) \\
& +217680 K^{6} q^{2} \cosh (6 K x) \\
& -20 K^{2} P^{2} \cosh (8 K x) \\
& \text { - } 928 K^{4} P q \cosh (8 K x) \\
& -8000 K^{6} q^{2} \cosh (8 K x)
\end{aligned}
$$

$$
\begin{aligned}
& +K^{2} P^{2} \cosh (10 K x) \\
& +8 K^{4} P q \cosh (10 K x) \\
& +16 K^{6} q^{2} \cosh (6 K x) \\
& +592 a^{2} K P \sinh (2 K x) \\
& -86720 a^{2} K^{3} q \sinh (2 K x) \\
& +176 a^{2} K P \sinh (4 K x) \\
& +35264 a^{2} K^{3} q \sinh (4 K x) \\
& -80 a^{2} K P \sinh (6 K x) \\
& \left.-2624 a^{2} K^{3} q \sinh (6 K x)\right) \\
& -2 a^{2} K^{2} t^{2 \alpha} \Gamma(0.5+\alpha)
\end{aligned}
$$$$
\times \Gamma(1+2 \alpha)(\operatorname{sech}(K x))^{4}
$$$$
\times\left(4^{3+\alpha} a^{4}-33 \times 2^{1+2 \alpha}(K P)^{2}+147\right.
$$$$
\left.\times 4^{2+\alpha} K^{4} P q-1113 \times 2^{5+2 \alpha} K^{6} q^{2}\right)
$$$$
-2^{1+2 \alpha}\left(\left(32 a^{4}+39(K P)^{2}-744 K^{4} P q\right.\right.
$$$$
\left.-36000 K^{6} q^{2}\right)
$$$$
x \cosh (2 K x)+3
$$$$
\times 2^{9+2 \alpha} K^{4} q
$$$$
\times\left(-P+20 K^{2} q\right) \cosh (4 K x)
$$$$
+15 \times 4^{\alpha} K^{2} P^{2} \cosh (6 K x)-57
$$$$
\times 2^{3+2 \alpha} K^{4} P q \cosh (6 K x)-705
$$$$
\times 4^{2+\alpha} K^{6} q^{2} \cosh (6 K x)+2^{1+3 \alpha}
$$$$
\times K^{2} P^{2} \cosh (8 K x)+13 \times 4^{2+\alpha}
$$$$
\times K^{4} P q \cosh (8 K x)+25 \times 2^{5+2 \alpha}
$$$$
\times K^{6} q^{2} \cosh (8 K x)
$$$$
-4^{\alpha} K^{2} P^{2} \cosh (10 K x)
$$$$
-2^{3+2 \alpha} K^{4} P q \cosh (10 K x)
$$$$
-4^{2+\alpha} K^{6} q^{2} \cosh (10 K x)+7
$$$$
\times 4^{2+\alpha} a^{2} K P \sinh (2 K x)+65
$$$$
\times 4^{3+\alpha} a^{2} K^{3} q \sinh (2 K x)-2^{5+2 \alpha} a^{2}
$$$$
\times K P \sinh (4 K x)-13 \times 2^{7+2 \alpha} a^{2} K^{3}
$$

$\times q \sinh (4 K x)$ 


$$
\begin{aligned}
& +4^{2+\alpha} a^{2} K q \sinh (6 K x) \\
& \left.+4^{3+\alpha} a^{2} K^{3} q \sinh (6 K x)\right) \\
& \times \tanh (K x)))
\end{aligned}
$$

Remark 8. It worth noting that, in this investigation, both techniques have provided the same results. However, from their methodologies one can observes that the HDM is very easy to implement and the complexity of the HDM is of order $n$.

\section{Conclusion}

We derived approximated solutions of nonlinear fractional Kawahara equations using comparatively innovative analytical modus operandi, the HDM and STM. We offered the epigrammatic history and some properties of fractional derivative concept. It is established that HDM and STM are authoritative and well-organized instruments of FPDEs. Additionally, the calculations concerned are very simple and uncomplicated.

\section{Conflict of Interests}

All authors declare there is no conflict of interests for this paper.

\section{Authors' Contribution}

The first draft was written by Abdon Atangana and Necdet Bildik, and the revised form was corrected in detail by S. C. Noutchie. All authors read and submitted the last version.

\section{Acknowledgments}

The first and the second authors would like to thank the third author for the time used to check all the kind of plagiarism and uncorrected mathematics. All authors would like to thank the reviewers for their comments.

\section{References}

[1] K. B. Oldham and J. Spanier, The Fractional Calculus, Academic Press, New York, NY, USA, 1974.

[2] I. Podlubny, Fractional Differential Equations, vol. 198 of Mathematics in Science and Engineering, Academic Press, New York, NY, USA, 1999.

[3] A. A. Kilbas, H. M. Srivastava, and J. J. Trujillo, Theory and Applications of Fractional Differential Equations, vol. 204, Elsevier, Amsterdam, , The Netherlands, 2006.

[4] M. Kurulay, B. A. IbrahimoǧF;lu, and M. Bayram, "Solving a system of nonlinear fractional partial differential equations using three dimensional differential transform method," International Journal of Physical Sciences, vol. 5, no. 6, pp. 906-912, 2010.

[5] M. Caputo, "Linear models of dissipation whose Q is almost frequency independent-part II," Geophysical Journal International, vol. 13, no. 5, pp. 529-539, 1967.
[6] A. A. Kilbas, H. M. Srivastava, and J. J. Trujillo, Theory and Applications of Fractional Differential Equations, vol. 204, Elsevier, Amsterdam, , The Netherlands, 2006.

[7] J. F. Botha and A. H. Cloot, "A generalised groundwater flow equation using the concept of non-integer order derivatives," Water SA, vol. 32, no. 1, pp. 1-7, 2006.

[8] A. Kilicman and Z. A. A. Al Zhour, "Kronecker operational matrices for fractional calculus and some applications," Applied Mathematics and Computation, vol. 187, no. 1, pp. 250-265, 2007.

[9] G. M. Zaslavsky, Hamiltonian Chaos and Fractional Dynamics, Oxford University Press, Oxford, UK, 2005.

[10] A. Atangana and J. F. Botha, "A generalized groundwater flow equation using the concept of variable-order derivative," Boundary Value Problems, vol. 2013, article 53, 2013.

[11] M. Wadati, "Introduction to solitons," Pramana, vol. 57, no. 5-6, pp. 841-847, 2001.

[12] M. J. Ablowitz and H. Segur, Solitons and the Inverse Scattering Transform, vol. 4 of SIAM Studies in Applied Mathematics, SIAM, Philadelphia, Pa, USA, 1981.

[13] W. Malfliet, "Solitary wave solutions of nonlinear wave equations," The American Journal of Physics, vol. 60, no. 7, pp. 650654, 1992.

[14] R. Hirota, "Direct method of finding exact solutions of nonlinear evolution equations," in Backlund Transformations, R. Bullough and P. Caudrey, Eds., pp. 1157-1175, Springer, Berlin, Germany, 1980.

[15] W. Malfliet and W. Hereman, "The tanh method-I: exact solutions of nonlinear evolution and wave equations," Physica Scripta, vol. 54, no. 6, pp. 563-568, 1996.

[16] E. Fan, "Extended tanh-function method and its applications to nonlinear equations," Physics Letters A, vol. 277, no. 4-5, pp. 212-218, 2000.

[17] J.-H. He and X.-H. Wu, "Exp-function method for nonlinear wave equations," Chaos, Solitons \& Fractals, vol. 30, no. 3, pp. 700-708, 2006.

[18] J. H. He and M. A. Abdou, "New periodic solutions for nonlinear evolution equations using Exp-function method," Chaos, Solitons \& Fractals, vol. 34, no. 5, pp. 1421-1429, 2007.

[19] X.-H. Wu and J.-H. He, "EXP-function method and its application to nonlinear equations," Chaos, Solitons \& Fractals, vol. 38, no. 3, pp. 903-910, 2008.

[20] A. M. Wazwaz, "The tanh and the sine-cosine methods for a reliable treatment of the modified equal width equation and its variants," Communications in Nonlinear Science and Numerical Simulation, vol. 11, no. 2, pp. 148-160, 2006.

[21] A. Atangana, "New class of Boundary value problems," Information Sciences Letters, vol. 1, no. 2, pp. 67-76, 2012.

[22] B. Zheng, "Exp-function method for solving fractional partial differential equations," The Scientific World Journal, vol. 2013, Article ID 465723, 8 pages, 2013.

[23] E. Hesameddini and H. Latifizadeh, "Reconstruction of variational iteration algorithm using the Laplace transform," Iternational Journal of Nonlinear Sciences and Numerical Simulation, vol. 10, pp. 1365-1370, 2009.

[24] E. Hesameddini and A. Rahimi, "A novel iterative method for solving systems of fractional differential equations," Journal of Applied Mathematics, vol. 2013, Article ID 428090, 7 pages, 2013.

[25] K. S. Miller and B. Ross, An Introduction to the Fractional Calculus and Fractional Differential Equations, A Wiley-Interscience Publication, John Wiley \& Sons, New York, NY, USA, 1993. 
[26] I. Podlubny, Fractional Differential Equations, vol. 198 of Mathematics in Science and Engineering, Academic Press, San Diego, CA, 1999.

[27] S. G. Samko, A. A. Kilbas, and O. I. Marichev, Fractional Integrals and Derivatives, Gordon and Breach Science, Yverdon, Switzerland, 1993.

[28] G. Jumarie, "On the representation of fractional Brownian motion as an integral with respect to $(\mathrm{d} t)^{a}$," Applied Mathematics Letters, vol. 18, no. 7, pp. 739-748, 2005.

[29] G. Jumarie, "Modified Riemann-Liouville derivative and fractional Taylor series of nondifferentiable functions further results," Computers \& Mathematics with Applications, vol. 51, no. 9-10, pp. 1367-1376, 2006.

[30] A. Atangana and A. Secer, "The time-fractional coupledKorteweg-de-Vries equations," Abstract and Applied Analysis, vol. 2013, Article ID 947986, 8 pages, 2013.

[31] A. Atangana, O. A. Ahmed, and N. Bıldık, "A generalized version of a low velocity impact between a rigid sphere and a transversely isotropic strain-hardening plate supported by a rigid substrate using the concept of noninteger derivatives," Abstract and Applied Analysis, vol. 2013, Article ID 671321, 9 pages, 2013.

[32] A. Atangana and E. Alabaraoye, "Solving a system of fractional partial differential equations arising in the model of HIV infection of $\mathrm{CD}^{+}$cells and attractor one-dimensional KellerSegel equations," Advances in Difference Equations, vol. 2013, article 94, 2013.

[33] A. Atangana and N. Bildik, "Approximate solution of tuberculosis disease population dynamics model," Abstract and Applied Analysis, vol. 2013, Article ID 759801, 8 pages, 2013.

[34] G. K. Watugala, "Sumudu transform: a new integral transform to solve differential equations and control engineering problems," International Journal of Mathematical Education in Science and Technology, vol. 24, no. 1, pp. 35-43, 1993.

[35] A. Atangana and D. Baleanu, "Nonlinear fractional JaulentMiodek and Whitham-Broer-Kaup equations within Sumudu transform," Abstract and Applied Analysis, vol. 2013, Article ID 160681, 8 pages, 2013.

[36] G. K. Watugala, "Sumudu transform: a new integral transform to solve differential equations and control engineering problems," International Journal of Mathematical Education in Science and Technology, vol. 24, no. 1, pp. 35-43, 1993.

[37] S. Weerakoon, "Application of Sumudu transform to partial differential equations," International Journal of Mathematical Education in Science and Technology, vol. 25, no. 2, pp. 277-283, 1994.

[38] Y. Nawaz, "Variational iteration method and homotopy perturbation method for fourth-order fractional integro-differential equations," Computers \& Mathematics with Applications, vol. 61, no. 8, pp. 2330-2341, 2011.

[39] H. Eltayeb and A. Kilıçman, "A note on the Sumudu transforms and differential equations," Applied Mathematical Sciences, vol. 4, no. 21-24, pp. 1089-1098, 2010.

[40] V. G. Gupta and B. Sharma, "Application of Sumudu transform in reaction-diffusion systems and nonlinear waves," Applied Mathematical Sciences, vol. 4, no. 9-12, pp. 435-446, 2010.

[41] A. Atangana and A. Kılıçman, "The use of Sumudu transform for solving certain nonlinear fractional heat-like equations," Abstract and Applied Analysis, vol. 2013, Article ID 737481, 12 pages, 2013. 


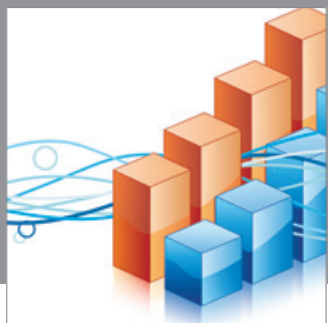

Advances in

Operations Research

mansans

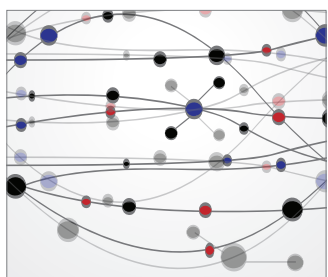

The Scientific World Journal
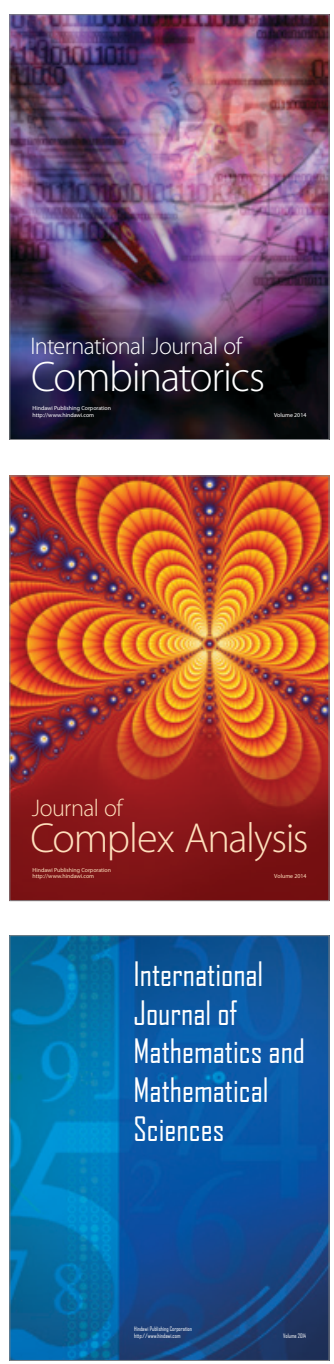
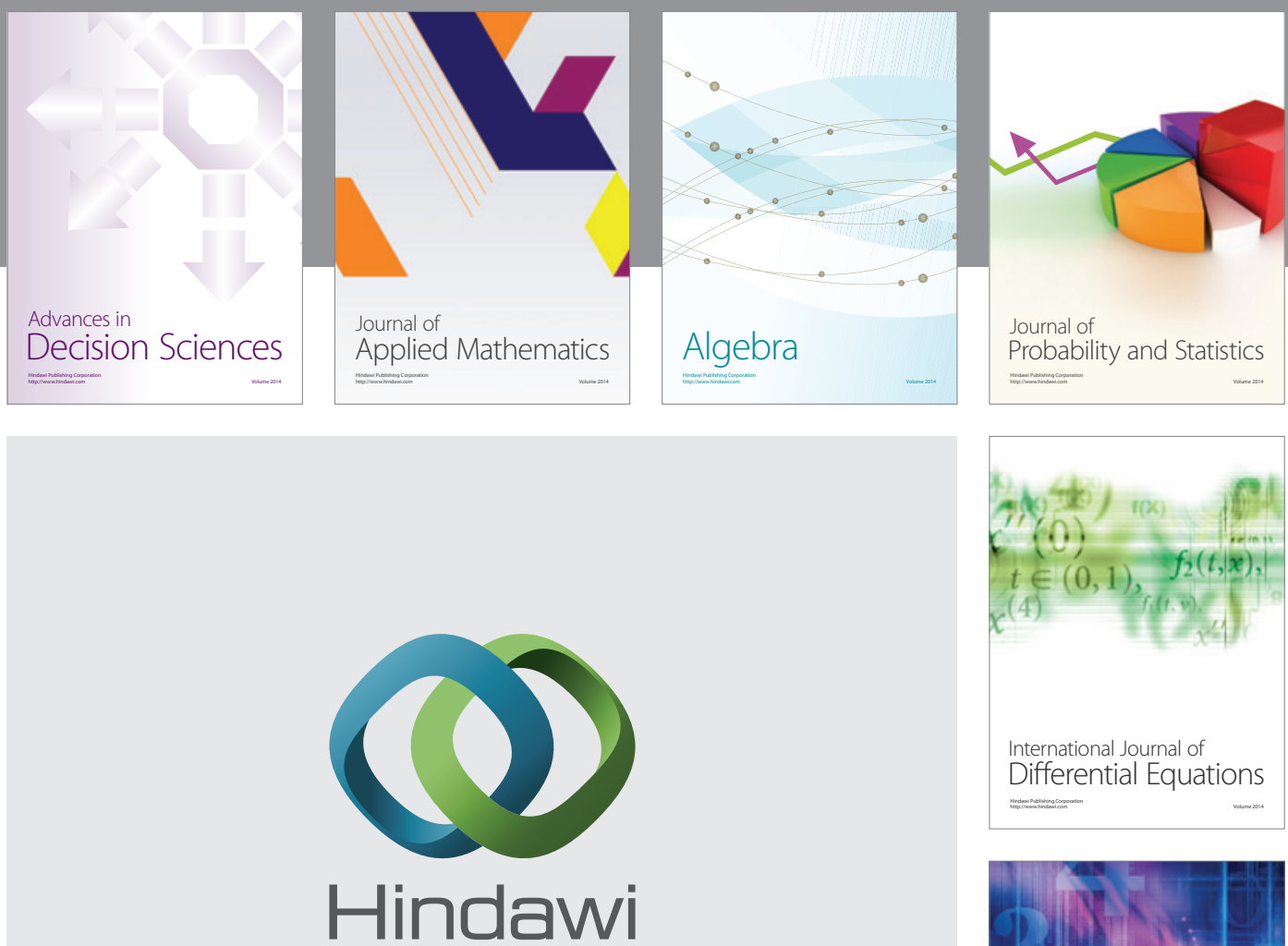

Submit your manuscripts at http://www.hindawi.com
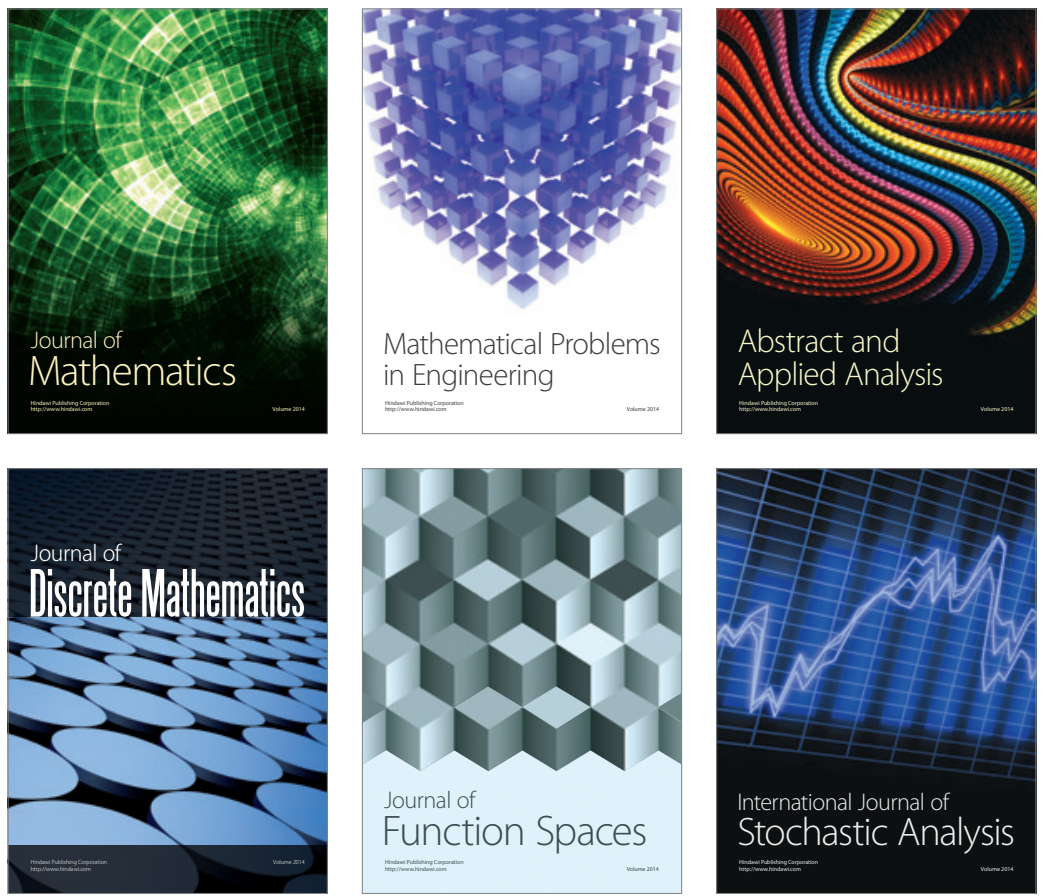

Journal of

Function Spaces

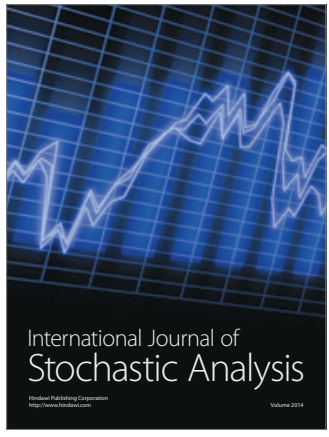

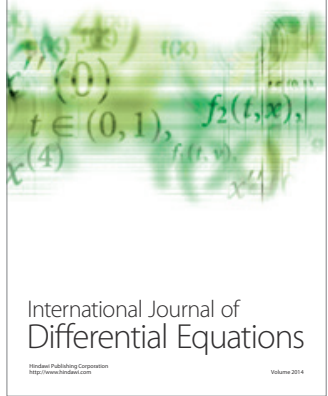
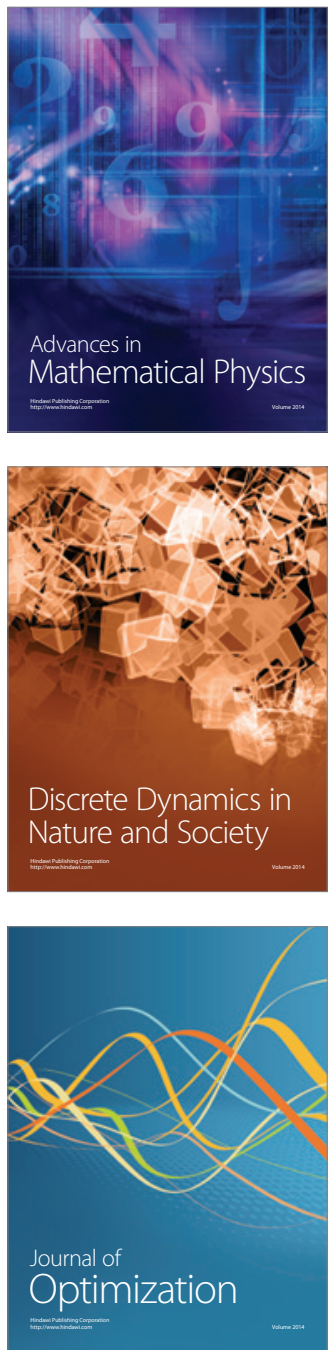